\title{
Hartz sei Dank? Stabilität und Entloh- nung neuer Jobs nach Arbeitslosigkeit
}

\author{
An den Hartz-Reformen scheiden sich die Geister. Positiven Entwicklungen wie sinken- \\ der Arbeitslosigkeit und steigender Beschäftigung stehen negative Aspekte wie mehr \\ prekäre Beschäftigung und größere Lohnspreizung entgegen. Ziel dieses Artikels ist es, \\ vermeintliche Verbesserungen des Arbeitsmarkts kritisch zu hinterfragen und die Ver- \\ änderung der Beschäftigungsqualität in den Jahren 1998 bis $2010 \mathrm{zu}$ analysieren. Haben \\ sich die Entlohnung und Beschäftigungsstabilität im Anschluss an eine Arbeitslosigkeit \\ seit Einführung der Hartz-Reformen verändert? Sind Langzeitarbeitslose besonders von \\ negativen Auswirkungen betroffen?
}

URSULA JAENICHEN, THOMAS ROTHE

\section{Einleitung}

Hohe und vor allem persistente Arbeitslosigkeit waren in den 1980er und 90er Jahren typische Kennzeichen des deutschen Arbeitsmarkts. Deshalb zielten die Hartz-Reformen (vgl. Knuth/Kaps in diesem Heft) nicht nur darauf, die Arbeitslosigkeit zu reduzieren und den Beschäftigungsstand zu erhöhen. Angestrebt wurde vielmehr auch, die Funktionsfähigkeit des Arbeitsmarkts zu verbessern, indem Sucharbeitslosigkeit verringert und somit die Arbeitslosigkeitsdauern insgesamt verkürzt würden. Die Neuorganisation der Bundesanstalt für Arbeit zu einem modernen Dienstleister bildete einen zentralen Punkt der Reformen. Eine effizient ausgestaltete Arbeitsvermittlung, freier Marktzutritt für private Arbeitsvermittler, intensivere Aktivierung Arbeitsloser und mehr Eigenverantwortung bei der Arbeitsuche sollten die Anreize für eine rasche Arbeitsaufnahme erhöhen. Außerdem sollte die Qualität der Vermittlung verbessert werden und somit die Arbeitslosigkeit und der verfestigte Kern der Langzeitarbeitslosigkeit abgebaut werden.

Veränderte Institutionen am Arbeitsmarkt, schärfere Sanktionsmöglichkeiten durch die Arbeitsagenturen und Jobcenter und vor allem deutlich geringere Lohnersatzleistungen im Falle längerer Arbeitslosigkeit könnten sich, so der Ausgangspunkt für die vorliegende Untersuchung, auf die Qualität neu begonnener Beschäftigungsverhältnisse auswirken. Dabei ist mit sinkenden Reservationslöhnen zu rechnen, weil Arbeits- lose wegen dieser Veränderungen eher als vor den Reformen bereit sein werden, zu geringeren Löhnen eine Beschäftigung aufzunehmen, um möglichst schnell die Arbeitslosigkeit zu verlassen und so Langzeitarbeitslosigkeit zu vermeiden. Durch die Hartz-Reformen sollte der Arbeitsmarkt auch generell flexibler werden. Insofern lässt sich einerseits vermuten, dass die Beschäftigungsstabilität während und nach den HartzReformen zurückgegangen ist. Andererseits kann argumentiert werden, dass Beschäftigte, darunter auch vormals arbeitslose Personen, seltener von sich aus kündigen, entweder weil sie die neue Beschäftigung für nicht attraktiv genug halten, oder um das Risiko erneuter und möglicherweise lang anhaltender Arbeitslosigkeit und damit verbundenen sozialen $\mathrm{Ab}$ stiegs zu minimieren. Dieses Verhalten würde zu längeren Beschäftigungsdauern führen.

Wir untersuchen die Stabilität und Entlohnung neu begonnener Beschäftigungsverhältnisse als bedeutende Dimensionen der Beschäftigungsqualität. Da Löhne in bestehenden Beschäftigungsverhältnissen in der Regel nur in extremen Krisensituationen nach unten angepasst werden, dürfte eine Veränderung des Lohnniveaus zunächst in den Einstiegslöhnen sichtbar werden. Die Betrachtung von Personen, die aus Arbeitslosigkeit in Beschäftigung gewechselt sind, rechtfertigt sich aus den Inhalten der Hartz-Reformen, die im Wesentlichen auf den Abbau von Arbeitslosigkeit gerichtet sind. ${ }^{1}$ Darüber hinaus kann vermutet werden, dass arbeitslose Personen bei Aufnahme einer neuen Beschäftigung in einer schwächeren Verhandlungsposition 
ABB. 1

\section{Abgangsraten aus Arbeitslosigkeit in Beschäftigung auf dem ersten Arbeitsmarkt nach Dauer vorangegangener Arbeitslosigkeit}

Angaben in Prozent

$\begin{array}{lll}- \text { bis } 6 \text { Monate } & 6 \text { bis } 9 \text { Monate } & 2 \text { Jahre u. länger }\end{array} \quad$ bis unter 2 Jahre $\quad-9$ bis 12 Monate

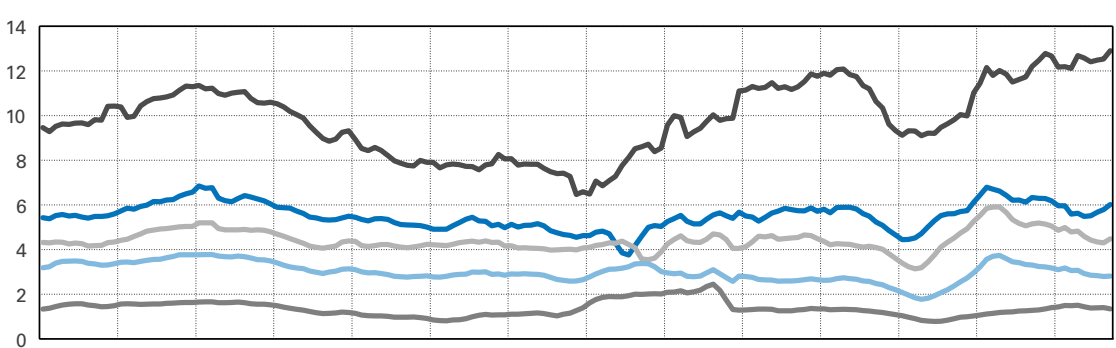

Apr 98 Apr 99 Apr00 Apr01 Apr02 Apr03 Apr04 Apr05 Apr06 Apr07 Apr08 Apr09 Apr 10 Apr 11

Anmerkung: Arbeitslose zwischen 25 und 54 Jahren, gleitender Dreimonatsdurchschnitt saisonbereinigter Monatswerte.

Quelle: Bundesagentur für Arbeit, ohne Daten der zugelassenen kommunalen Träger; Berechnungen der Autoren. monatlichen Abgangsraten aus Arbeitslosigkeit (Abbildung 1). Die Abgangsraten sind definiert als Anteil der Arbeitslosen einer Dauerkategorie, die eine sozialversicherungspflichtige Beschäftigung am ersten Arbeitsmarkt aufnehmen, an allen Arbeitslosen mit gleicher Dauer der Arbeitslosigkeit.

Die Abgangsraten in Beschäftigung sinken mit zunehmender Dauer der Arbeitslosigkeit. Darüber hinaus lässt sich ab dem Jahr 2005 ein deutlicher Anstieg der Abgangsraten bei Personen mit Arbeitslosigkeitsdauern unter sechs Monaten erkennen. Im Jahr 2000 hatten Kurzzeitarbeitslose im Vergleich zu Personen mit über zwei Jahren Arbeitslosigkeit eine etwa fünfmal höhere Beschäftigungschance. Im Jahr 2011 war die Abgangsrate aus Kurzzeitarbeitslosigkeit in Beschäftigung sogar achtmal höher als aus Langzeitarbeitslosigkeit.

Für die Jahre 2000 bis 2003, den Zeitraum unmittelbar vor den Hartz-Reformen, zeigen sich sinkende Abgangsraten quer über alle Dauerkategorien. Zwischen dem Jahr 2005 und dem Jahr 2007, bei Langzeitarbeitslosen mit Dauern über zwei Jahren bereits etwas früher, steigen die Abgangsraten für alle Dauergruppen zumindest temporär an. Danach zeigt sich ein paralleler, aber eher kurzfristiger Einbruch der Abgangsraten in allen Gruppen während der Rezession 2008/2009.

Insgesamt ist außer bei Kurzzeitarbeitslosen auch bei Arbeitslosen mit mittleren Dauern eine leicht positive Entwicklung der Abgangsraten nach 2004 festzustellen. Besonders für Arbeitslose mit Dauern zwischen neun und zwölf Monaten wäre jedoch ein stärkerer Anstieg der Abgangsrate zu erwarten gewesen, da sich der Anreiz zur Beschäftigungsaufnahme vor Auslaufen des Anspruchs auf Arbeitslosengeld ab Januar 2005 deutlich erhöht haben müsste.

Rusammen, um zu zeigen, welche Effekte institutionellen Änderungen auf Arbeitslosigkeit, Beschäftigung und die generelle Funktionsfähigkeit des Arbeitsmarkts ausgegangen sind (3).

Anschließend untersuchen wir, inwieweit sich die Beschäftigungsqualität in neu aufgenommenen Beschäftigungsverhältnissen im Anschluss an eine Arbeitslosigkeitsphase in Westdeutschland im Zeitraum von 1998 bis 2009/2010 verändert hat (4). Hier beginnen wir mit einem Überblick über die Lohnverteilung und einer Untersuchung der Löhne vorher arbeitsloser Personen unter Berücksichtigung individueller, betrieblicher und regionaler Einflussfaktoren (4.2). Danach werden ähnliche Analysen für die Beschäftigungsdauern durchgeführt (4.3). Eine kritische Würdigung der Ergebnisse rundet den Artikel ab (5).

\section{Abgänge aus Arbeitslosigkeit in Beschäftigung}

Einen Eindruck davon, wie sich die Situation von Arbeitslosen seit 1998 in Westdeutschland verändert hat, geben die

\section{Wirkung der Hartz-Reformen auf den Arbeitsmarkt}

Die positive Entwicklung des Arbeitsmarkts seit 2005 wird sowohl auf die spezifischen institutionellen Bedingungen in Deutschland als auch auf deren Veränderung durch die Hartz-Reformen zurückgeführt oder damit in engem $\mathrm{Zu}-$ sammenhang gesehen (vgl. Klinger et al. 2013). Seither hat sich die Arbeitslosigkeit halbiert und die sozialversicherungspflichtige Beschäftigung ist um etwa zwei Millionen Personen gestiegen. Darüber hinaus hatte die Rezession 2008/2009 nur geringe Auswirkungen auf den deutschen Arbeitsmarkt, was zumindest teilweise mit den Strukturreformen erklärt werden kann (vgl. Burda/Hunt 2011;

1 Weitere Analysen zu Entlohnung und Beschäftigungsdaue in neuen Beschäftigungsverhältnissen finden sich in Giannelli et al. (2013). 
Möller 2010). Gartner/Klinger (2010) argumentieren, dass die Krisenfestigkeit des deutschen Arbeitsmarkts, das „Beschäftigungswunder", neben der moderaten Lohnentwicklung auch auf institutionelle Änderungen im Zuge der Hartz-Reformen zurückzuführen sei.

Mehrere Studien untersuchen die Veränderung der Funktionsfähigkeit des deutschen Arbeitsmarktes nach den Hartz-Reformen anhand empirischer Matchingfunktionen. Fahr/Sunde (2009) sowie Klinger/Rothe (2012) verwenden Individualdaten des Instituts für Arbeitsmarktund Berufsforschung (IAB) und können eine verbesserte Matching-Effizienz seit den Jahren 2003 und 2004 nachweisen, also ein schnelleres Zusammenkommen von $\mathrm{Ar}$ beitslosen und offenen Stellen. Eine Studie von Hertweck/ Sigrist (2012) auf Basis des Sozio-oekonomischen Panels (SOEP) konzentriert sich auf die Hartz-IV-Reform im Jahr 2005 und zeigt ebenfalls beschleunigte Übergänge aus Arbeitslosigkeit in Beschäftigung. Obwohl diese Studien keinen direkten Wirkungszusammenhang analysieren können, zeigen sie doch, dass die Matching-Effizienz in den Jahren 2003 bis 2005 im Vergleich zu den Vorjahren gestiegen ist. Demnach haben sich die Chancen von Arbeitslosen, in Beschäftigung zu kommen, unabhängig von der konjunkturellen Situation deutlich verbessert.

Die Ergebnisse einer repräsentativen Betriebsbefragung verweisen auf ein verändertes Suchverhalten von Bewerbern nach den Arbeitsmarktreformen. Rebien/Kettner (2011) finden empirische Belege für eine gestiegene Konzessionsbereitschaft sowohl von arbeitslosen Bewerbern bei Aufnahme einer neuen Beschäftigung als auch innerhalb der Belegschaft aus Sicht der befragten Betriebe. Fast ein Drittel von ihnen gab an, dass die Bereitschaft, eine unterwertige Stelle anzunehmen, ungünstige Arbeitsbedingungen wie Schichtarbeit oder eine größere Entfernung zum Arbeitsort zu akzeptieren oder Zugeständnisse bei der Entlohnung zu machen, im Jahr 2005 gegenüber 2004 gestiegen sei.

Hinsichtlich der Beschäftigungsstabilität zeigt Rothe (2009), dass im konjunkturellen Aufschwung nach den Hartz-Reformen wesentlich weniger Beschäftigungen begonnen und beendet wurden als im vorangegangenen Aufschwung. Das bedeutet im Umkehrschluss, dass die bestehenden Beschäftigungsverhältnisse in den Jahren 2006 bis 2008 von längerer Dauer waren als zwischen 1998 und 2000. Erlinghagen (2010) verweist diesbezüglich auf „die Diskrepanz zwischen, gefühlter` Beschäftigungsunsicherheit und realer Beschäftigungsstabilität" und führt die Entwicklung neben der zunehmenden Einkommensungleichheit auch auf den drohenden Statusverlust im Falle lang anhaltender Arbeitslosigkeit zurück (siehe auch Bothfeld/Rosenthal in diesem Heft).

Untersuchungen von Koller/Rudolph (2011) zeigen im Kontrast dazu, dass Arbeitsaufnahmen von ALG-II-Leistungsempfängern häufig von kurzer Dauer sind: Nur $55 \%$ der begonnenen Beschäftigungsverhältnisse im Jahr 2008 dauerten länger als sechs Monate. Die Gefahr von Drehtüreffekten, dem ständigen Wechsel zwischen Arbeitslosigkeit und kurzen Phasen von Erwerbstätigkeit, ist vor allem für Geringqualifizierte groß. Darüber hinaus bezog etwa die Hälfte der Personen, die aus dem ALG-II-Bezug heraus eine Beschäftigung aufnahmen, weiterhin - wenn auch reduzierte - Leistungen nach dem SGB II. Etwa zwei Drittel der erwerbstätigen Leistungsbezieher im SGB II, der sogenannten Aufstocker, gelang es allerdings, im Jahr 2008 den Leistungsbezug zu beenden (siehe auch Rudolph in diesem Heft).

Ein weiterer Literaturstrang quantifiziert gesamtwirtschaftliche Effekte der Hartz-Reformen auf Basis theoretisch fundierter makroökonomischer Gleichgewichtsmodelle. Die Modelle dienen der Schätzung und Simulation quantitativer Einflüsse der Hartz-Reformen auf Arbeitslosigkeit, Wachstum und Wohlfahrt. Die so ermittelten Effekte lassen sich unter Berücksichtigung der getroffenen Annahmen kausal interpretieren. Allerdings variieren die Ergebnisse verschiedener Studien beträchtlich, was hauptsächlich auf die unterschiedliche Berücksichtigung der Neuregelungen der Arbeitslosenunterstützung in den Modellen zurückzuführen sein dürfte, wie Launov/Wälde (2013a) betonen. ${ }^{2}$ Während Krause/Uhlig (2012) rund drei Viertel des Rückgangs der gleichgewichtigen Arbeitslosigkeit zwischen 2005 und 2008 mit der Einführung von Hartz IV erklären, sind die Effekte im Modell von Krebs/ Scheffel (2013) nur etwa halb so groß und Launov/Wälde (2013b) finden keine nennenswerten Effekte der HartzIV-Reform. In einer neueren Studie erweitern Launov/ Wälde (2013a) ihr Modell auf die gesamte Arbeitsmarktreform und kommen zu dem Schluss, dass eine effizientere Arbeitsvermittlung als Kernelement von Hartz III rund ein Drittel des Rückgangs der gleichgewichtigen Arbeitslosigkeit begründet, während die Einführung von Hartz IV nur $7 \%$, d. h. 0,3 Prozentpunkte, erklärt.

Positive Wohlfahrtseffekte werden in den genannten Makromodellen insbesondere von Beschäftigten und Kurzzeitarbeitslosen realisiert (vgl. Krebs/Scheffel 2013), weil für diese Gruppe positive Anreizeffekte zur Arbeitsaufnahme und in der Folge kürzere Arbeitslosigkeitsdauern den Rückgang der Versicherungsleistung im Falle längerer Arbeitslosigkeit überkompensieren. Beschäftigte profitieren darüber hinaus von der Reduktion des Beitragssatzes zur Arbeitslosenversicherung. Da Langzeitarbeitslose durch die Einführung des im Vergleich zur Arbeitslosenhilfe reduzierten Arbeitslosengelds II Wohlfahrtsverluste hinnehmen müssen, werden bestehende Ungleichheiten auf dem Arbeitsmarkt durch die Hartz-Reformen tendenziell eher verfestigt als beseitigt.

2 Die Einkommenssituation von Haushalten mit gering qualifizierten Arbeitslosen hat sich z. T. durch die Einführung von Hartz IV sogar verbessert (Goebel/Richter 2007). Wird das nicht berücksichtigt, sondern im Modell ein stark sinkendes Haushaltseinkommen angenommen (wie etwa bei Krause/Uhlig 2012), führt das zu höheren Arbeitsanreizen und erklärt folglich einen größeren Teil des Rückgangs der Arbeitslosigkeit (vgl. Launov/Wälde 2013a). 
Die Motivation für die hier vorgelegte Untersuchung besteht im Wesentlichen in der Frage, ob sich die geänderten institutionellen Rahmenbedingungen, insbesondere die von den Hartz-Reformen beabsichtigte Beschleunigung der Arbeitsaufnahme, auf die Entlohnung und Beschäftigungsstabilität von zuvor arbeitslosen Personen ausgewirkt haben. Unsere Datenbasis erlaubt uns dabei sowohl einen Blick auf die Verteilung der Löhne und Beschäftigungsdauern in neuen Beschäftigungsverhältnissen als auch auf den Einfluss unterschiedlicher Merkmale wie Arbeitslosigkeitsdauer, Qualifikationsniveau und Wirtschaftszweig. Für Personen mit vorheriger Arbeitslosigkeit werden verschiedene Einflussfaktoren auf Einstiegslöhne und Beschäftigungsdauern untersucht, wobei durch die Abgrenzung von Teilzeiträumen ein möglicher Einfluss des Reformzeitraums und des Zeitraums danach sichtbar gemacht wird.

\section{Ergebnisse auf Basis von Individual- daten}

Wir gehen im Folgenden der Frage nach, ob sich im Zuge der Hartz-Reformen die Qualität neuer, im Anschluss an eine Arbeitslosigkeitsphase aufgenommener Beschäftigungsverhältnisse verändert hat und welche Unterschiede diesbezüglich zwischen Personen mit unterschiedlichen Dauern der Arbeitslosigkeit bestehen.

Vorweggeschickt sei, dass es sich nicht um eine kausale Analyse handelt. Da die Hartz-Reformen auf den gesamten Arbeitsmarkt gewirkt haben, existiert keine Kontroll- oder Vergleichsbeobachtung für Ergebnisse, die ohne die Reformen erzielt worden wären. Im speziellen Fall bedeutet dies, dass die beobachteten Löhne und Beschäftigungsdauern auch von der Globalisierung, der Konjunktur oder sonstigen Faktoren beeinflusst sind, ohne dass sich exakt ein „HartzEffekt" isolieren ließe.

\subsection{Datenbasis: Integrierte Erwerbsbiografien}

Für die Analyse von Löhnen und Beschäftigungsstabilität verwenden wir einen großen repräsentativen Individualdatensatz des IAB, die Integrierten Erwerbsbiografien (IEB). Wir verwenden eine Zwei-Prozent-Stichprobe und betrachten Übergänge aus Arbeitslosigkeit in sozialversicherungspflichtige Beschäftigung in Westdeutschland in den Jahren 1998 bis 2010. Da sich die Arbeitsmarktbedingungen in Ost- und Westdeutschland nach wie vor grundsätzlich unterscheiden, konzentrieren wir uns ausschließlich auf Westdeutschland und kontrollieren für zusätzliche regionale Effekte auf Ebene der Bundesländer.

Die Bruttolöhne und die Beschäftigungsdauern werden aus verpflichtenden Angaben berechnet, die vom Arbeitgeber an die Sozialversicherungsträger übermittelt werden. Die Beschäftigungsdauer ist definiert als Periode einer zusammenhängenden Beschäftigung bei einem Arbeitgeber. Unterbrechungen bis zu drei Monaten werden ignoriert. Bei den Lohnanalysen wird die Zensierung an der Beitragsbemessungsgrenze berücksichtigt.

Die beobachteten Löhne werden mit dem Verbraucherpreisindex deflationiert, um Reallöhne in Preisen von 2005 zu erhalten. Die Analyse der Löhne wird auf Vollzeitbeschäftigte beschränkt, weil uns keine Informationen über die genaue Arbeitszeit vorliegen und somit keine Stundenlöhne berechnet werden können. In den Analysen der Beschäftigungsdauer verbleiben Teilzeitbeschäftigte, sofern sie mehr als geringfügig beschäftigt sind. Personen in geringfügiger Beschäftigung und Minijobs, Auszubildende und Personen unter 25 und über 54 Jahren werden ausgeschlossen, um eine homogenere Stichprobe zu erhalten.

Wirtschaftszweige und Betriebsgrößenklassen gehen ebenso als Kontrollvariablen in die Schätzungen zur Lohnentwicklung und zur Beschäftigungsstabilität ein wie regionale Wachstumsraten des Bruttoinlandsprodukts aus der Volkswirtschaftlichen Gesamtrechnung der Länder und regionale Arbeitslosenquoten auf Ebene der 326 westdeutschen Kreise (NUTS 3). Saisonale Effekte werden durch Dummyvariablen für das jeweilige Quartal des Beschäftigungsbeginns berücksichtigt.

Darüber hinaus nutzen wir eine Reihe von soziodemografischen Merkmalen wie Alter, Nationalität, Berufserfahrung und Informationen zur schulischen und beruflichen Ausbildung. Für Männer und Frauen werden jeweils getrennte Modelle geschätzt, um ihrem unterschiedlichen Arbeitsmarktverhalten Rechnung zu tragen. Die Schätzungen zur Verweildauer beziehen sich auf Beschäftigungen mit Beginn in den Jahren 1998 bis 2008, damit Dauern von mindestens zwei Jahren untersucht werden können. Für die Lohnschätzungen nutzen wir aufgrund der Verfügbarkeit einzelner Kontrollvariablen die Jahre 1998 bis 2009.

\subsection{Lohnentwicklung}

Ergebnisse zur mittel- und langfristigen Entwicklung der Löhne und der Lohnungleichheit liegen aus mehreren Untersuchungen vor. Demnach hat - einhergehend mit einem Rückgang der Reallöhne bei neu aufgenommenen Beschäftigungsverhältnissen - die Lohnungleichheit in Deutschland zuletzt weiter zugenommen (vgl. Dustmann et al. 2009; Riphahn/Schnitzlein 2011; Giannelli et al. 2013).

Die Daten der IEB (Abbildung 2) belegen für die Medianlöhne in neu begonnenen Beschäftigungsverhältnissen insgesamt einen deutlichen Rückgang im Beobachtungszeitraum. Insbesondere bei Männern zeigt sich im Zeitraum von 2001 bis 2006 ein Rückgang um rund $12 \%$. Die Medianlöhne der Frauen insgesamt sanken in dieser Zeit zwar auch, jedoch nur um etwa $8 \%$, sodass sich die Einstiegslöhne von Männern und Frauen etwas annäherten. Der Abstand zwischen den Einstiegslöhnen aller von Männern neu begonnenen Beschäftigungen und den Einstiegslöhnen arbeitsloser Männer ist in diesem Zeitraum 
Veränderung der Abstände zwischen Quartilen der Lohnverteilung bei neu begonnener sozialversicherungspflichtiger Beschäftigung in Westdeutschland, 1998 - 2010

\begin{tabular}{lc|c|c|c}
\hline & \multicolumn{2}{c}{ Veränderung in Euro pro Tag } & \multicolumn{2}{c}{ Veränderung in Prozent } \\
& $\mathbf{5 0 - 2 5} \mathbf{\%}$ Perzentil & $\mathbf{7 5 - 5 0} \%$ Perzentil & $\mathbf{5 0 - 2 5} \mathbf{\%}$ Perzentil & $\mathbf{7 5 - 5 0} \%$ Perzentil \\
\hline Männer & 0,74 & 1,09 & 4,0 & 3,9 \\
\hline Frauen & 0,06 & 3,31 & 0,3 & 14,0 \\
\hline
\end{tabular}

Quelle: Integrierte Erwerbsbiografien (IEB), neu begonnene sozialversicherungspflichtige Vollzeitbeschäftigungsverhältnisse, Reallöhne in Preisen von 2005; Berechnungen der Autoren.

etwa stabil geblieben. ${ }^{3}$ Dagegen hat sich bei Frauen der Abstand in den Medianlöhnen von zuvor arbeitslosen Frauen und allen Frauen in neu begonnenen Beschäftigungsverhältnissen deutlich vergrößert. So lässt sich feststellen, dass der Rückgang der Reallöhne spätestens im Jahr 2002 eingesetzt hat und damit zeitlich den HartzReformen vorgelagert ist.

Einen weiteren Eindruck von Veränderungen in der Verteilung der Löhne in neu begonnenen Beschäftigungsverhältnissen (mit und ohne vorherige Arbeitslosigkeit) gibt Tabelle 1. Im Vergleich der Jahre 2010 und 1998 zeigt sich eine leichte Zunahme des Abstands zwischen dem unteren Lohnquartil und dem Medianlohn für Männer und Frauen (4,0 bzw. 0,3 \%). Der Lohnabstand zwischen den mittleren und oberen Einstiegslöhnen stieg für Männer und deutlich stärker für Frauen (3,9 bzw. 14,0 \%). Damit hat sich die Lohnungleichheit bei neu begonnenen Beschäftigungsverhältnissen für Frauen oberhalb des Medians deutlich erhöht, bei Männern ist sowohl bei den Einstiegslöhnen unterhalb des Medians als auch bei denen oberhalb des Medianlohns eine leichte Zunahme der Lohnspreizung festzustellen.

Im nächsten Schritt konzentrieren wir uns auf Personen, die nach einer Arbeitslosigkeitsphase eine Beschäftigung aufgenommen haben und schließen somit Job-to-JobWechsel und sonstige Übergänge aus. Gefragt wird danach, in welchem Umfang sich die in Abbildung 2 dargestellte Entwicklung der Einstiegslöhne im Zeitablauf auch dann zeigt, wenn in einem multivariaten Modell der Einfluss individueller, betrieblicher und regionaler Faktoren auf den Lohn berücksichtigt wird. Eine weitere Frage ist, ob die Dauer der Arbeitslosigkeit die Höhe des Einstiegslohns beeinflusst.

Es werden zensierte Regressionsmodelle ${ }^{4}$ geschätzt, um dem Fehlen von Lohndaten oberhalb der Beitragsbemessungsgrenze Rechnung zu tragen. Die abhängige Variable ist das logarithmierte Tagesentgelt.

Tabelle 2 enthält die zentralen Ergebnisse. Um den zeitlichen Zusammenhang zwischen den Hartz-Reformen und Veränderungen in den Einstiegslöhnen sichtbar zu machen, wird der Untersuchungszeitraum in die Periode vor den Hartz-Reformen (1998 - 2002), den Reform-
ABB. 2

\section{Entwicklung der Medianlöhne von Männern und Frauen in neuen Beschäftigungsverhältnissen in Westdeutschland 1998 - 2010*}

Angaben in Euro proTag
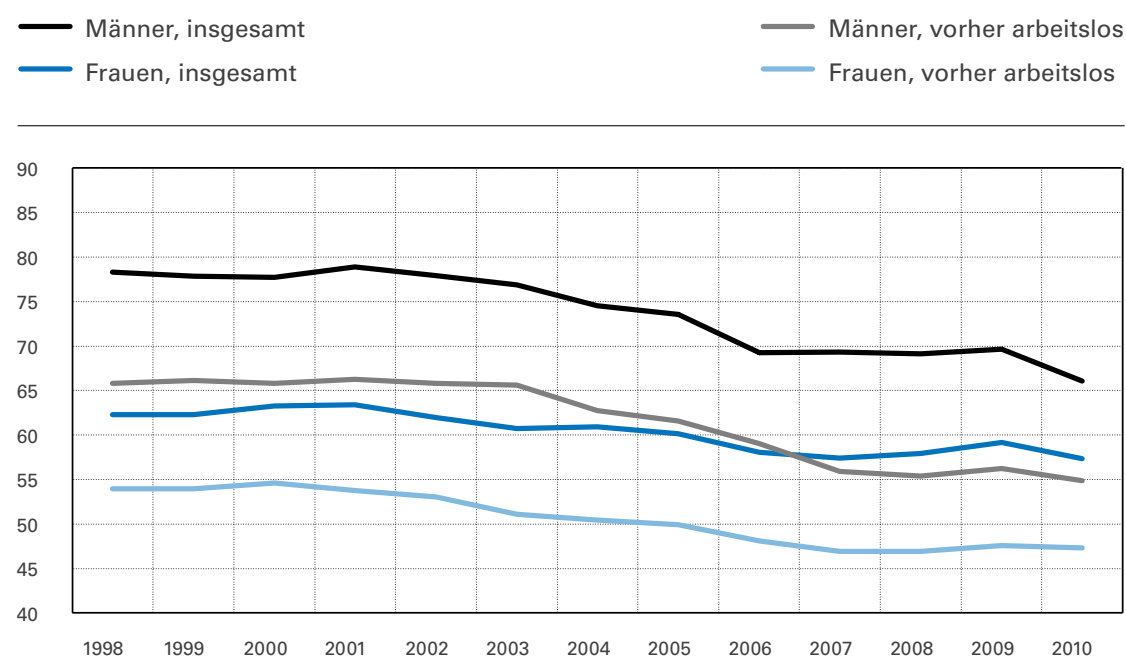

*insgesamt und nach Arbeitslosigkeit.

Anmerkung: Neu begonnene sozialversicherungspflichtige Vollzeitbeschäftigungsverhältnisse, Reallöhne in Preisen von 2005.

Quelle: IEB; Berechnungen der Autoren.

3 Der Anteil von Beschäftigungsverhältnissen, die im Anschluss an Arbeitslosigkeit neu begründet werden, an den neuen Beschäftigungsverhältnissen insgesamt liegt je nach Jahr und Gruppendefinition zwischen gut 20 und gut $30 \%$. Den größten Anteil mit 30 bis zu $45 \%$ nehmen Jobto-Job-Wechsel ein, bei denen die neue Beschäftigung ohne Unterbrechung nach einem anderen Job aufgenommen wird. Der Rest sind Beschäftigungen, bei denen die Personen zuvor längere Zeit nicht (oder noch nie) als beschäftigt oder arbeitslos registriert waren.

4 Das bekannteste zensierte Regressionsmodell ist das Tobit. Die hier geschätzten Modelle sind sehr ähnlich, Iassen jedoch eine über Beobachtungen veränderliche Zensierungsgrenze zu. 
TABELLE 2

Determinanten und Effekte unterschiedlicher Zeiträume auf Reallöhne in neu begonnenen Vollzeitbeschäftigungen nach Arbeitslosigkeit, Westdeutschland

\begin{tabular}{lcc}
\hline & Männer & Frauen \\
\hline Periode (Ref. 1998-2002) & & \\
\hline $2003-2005$ & $-0,024^{* * *}$ & $-0,050^{* * *}$ \\
$2006-2009$ & $-0,112^{* * *}$ & $-0,118^{* * *}$ \\
\hline
\end{tabular}

Dauer der Arbeitslosigkeit (Referenzkategorien bis 6 Monate)

\begin{tabular}{lcc}
\hline 6 bis 9 Monate & $-0,056^{* * *}$ & $-0,056^{* * *}$ \\
\hline 9 bis 12 Monate & $-0,091^{* *}$ & $-0,107^{* * *}$ \\
\hline 12 bis 24 Monate & $-0,127^{* * *}$ & $-0,140^{* * *}$ \\
\hline 24 Monate und mehr & $-0,194^{* * *}$ & $-0,173^{* * *}$ \\
\hline Konstante & $4,114^{* * *}$ & $3,911 * * *$ \\
\hline Anzahl der Beobachtungen & 71.922 & 35.568 \\
\hline
\end{tabular}

Weitere Kontrollvariablen: Qualifikation, potenzielle Berufserfahrung, Ausländer, Betriebsgröße, Wirtschaftszweig, regionale Arbeitsnachfrage, Bundesland.

Regressionskoeffizienten, Signifikanzniveau: * $10 \%, * * 5 \%$ und *** $1 \%$

Quelle: IEB; Statistisches Bundesamt; Berechnungen der Autoren.

zeitraum (2003-2005) und eine Nachreformperiode (2006 - 2009) unterteilt. Als Referenzperiode wird die Zeit vor den Hartz-Reformen gewählt und mit Dummyvariablen der Einfluss des Beginns der Beschäftigung in der Reform periode bzw. in der Nachreformperiode im Vergleich zur Referenzperiode 1998 bis 2002 geschätzt. Die Darstellung in Tabelle 2 beschränkt sich auf die hier im Mittelpunkt stehenden Schätzergebnisse für die Effekte der Perioden und die Arbeitslosigkeitsdauer, ausführliche Ergebnisse sind bei den Autoren erhältlich.

Als wichtigstes Ergebnis lässt sich festhalten, dass die Periodenvariablen durchweg signifikante und negative Effekte auf den Reallohn ausüben. In der Reformperiode 2003 bis 2005 ist der im Vergleich zum Zeitraum 1998 bis 2002 geschätzte Lohnrückgang noch mäßig (etwa $2 \%$ bei Männern und $5 \%$ bei Frauen), während für die Jahre nach der Reform sehr hohe Lohneinbußen von mehr als $10 \%$ bei Männern und Frauen geschätzt werden. ${ }^{5}$ Demnach sind auch bei Berücksichtigung der beobachteten individuellen, betrieblichen und regionalen Einflussfaktoren deutliche Lohneinbußen bei Aufnahme einer Beschäftigung nach Arbeitslosigkeit festzustellen. Der drastische Lohnrückgang in der letzten Periode kann jedoch nicht ausschließlich als Auswirkung der Hartz-Reformen angesehen werden, weil die vorliegenden Lohnschätzungen keine kausalen Interpretationen der Periodeneffekte erlauben. Vielmehr dürfte die Rezession 2008/2009 zumindest teilweise zum starken Lohnrückgang in den Jahren 2006 bis 2009 beigetragen haben.

Ein weiteres Ergebnis ist, dass mit zunehmender Dauer der vorangegangenen Arbeitslosigkeit in der neuen Beschäftigung deutlich geringere Löhne erzielt werden. Innerhalb der Gruppe zuvor arbeitsloser Personen werden für Perso- nen mit Arbeitslosigkeitsdauern von sechs bis neun Monaten Lohnabschläge von etwa $5 \%$ geschätzt, bei längeren Dauern betragen die Lohneinbußen oft weit über $10 \%$ und besonders bei langzeitarbeitslosen Männern und Frauen sind die Effekte sehr hoch.

Die bisher diskutierten Einflüsse bleiben im Wesentlichen erhalten, wenn alle erklärenden Variablen mit den Periodenindikatoren interagiert werden. Die Interaktionseffekte für langzeitarbeitslose Männer deuten während der Reformperiode auf geringere Einstiegslöhne hin. Für Frauen zeigt sich ein Lohnrückgang im Zeitraum nach den Hartz-Reformen bei Arbeitslosigkeitsdauern von über 24 Monaten.

Die Schätzergebnisse für die hier nicht in der Tabelle enthaltenen Kontrollvariablen entsprechen weitgehend den Erwartungen. Allerdings ist bemerkenswert, dass einzelne Merkmale zu relativ starken Lohnabschlägen führen. Einen positiven Einfluss auf den Lohn haben eine höhere Qualifikation, längere potenzielle Berufserfahrung (nur bei Männern), eine zunehmende Firmengröße sowie (nicht durchgängig) eine auf Kreisebene geringere Arbeitslosenquote und ein höheres Wirtschaftswachstum. Ausländern, Personen ohne Berufsausbildung und mit höchstens mittlerer Reife und Beschäftigte im Wirtschaftszweig „Persönliche und haushaltsbezogene Dienstleistungen“ erzielen signifikant niedrigere Löhne in der neuen Beschäftigung. Noch stärkere Abschläge gelten für die Einstiegslöhne in der Leiharbeitsbranche, bei Frauen im Vergleich zur Referenzbranche. Bei den Interaktionen der Kontrollvariablen mit den Periodenindikatoren sind die Effekte meist relativ gering und uneinheitlich.

\subsection{Beschäftigungsdauern}

Die Beschäftigungsdauer in neu begonnenen Beschäftigungsverhältnissen wird von uns als weiteres Merkmal der Qualität von Beschäftigungsverhältnissen angesehen, weil sie einerseits ein Maß für die Sicherheit des Arbeitsplatzes und andererseits ein Maß für die Möglichkeit zum Erwerb betriebsspezifischer Qualifikationen ist. Die mit den Hartz-Reformen getroffenen Regelungen zur Lockerung des Kündigungsschutzes und zur Erleichterung des Einsatzes von befristeter Beschäftigung oder Zeitarbeit sollten die Flexibilität auf dem Arbeitsmarkt erhöhen und somit eher auf den Abbau der Beschäftigungsstabilität zielen. Demnach wären zumindest für einen Teil der Beschäftigten kürzere Verweildauern im neuen Job zu erwarten als zuvor.

5 Die Koeffizienten von binären Variablen geben im loglinearen Modell ungefähr die prozentuale Differenz der abhängigen Variablen im Vergleich zur Referenzkategorie wieder. Bei größeren Werten der Koeffizienten (über 0,1) wird die Approximation jedoch zunehmend ungenau. 
Aus der aggregierten Betrachtung (Abbildung 3) lässt sich zunächst die Verbleibswahrscheinlichkeit der in den jeweiligen Jahren neu begonnenen Beschäftigungsverhältnisse $^{6}$ in Westdeutschland ablesen. Für alle männlichen Beschäftigten, die beispielsweise 1998 ein neues Beschäftigungsverhältnis begannen, lag die Wahrscheinlichkeit, nach sechs Monaten noch bei demselben Arbeitgeber beschäftigt zu sein, bei rund $72 \%$, für alle Frauen bei etwa $75 \%$. Allerdings waren die Beschäftigungsdauern bei zuvor arbeitslosen Personen deutlich kürzer. Bei Männern mit vorheriger Arbeitslosigkeit bestanden nach einem halben Jahr nur noch etwa 61 \% der im Jahr 1998 begonnenen Beschäftigungsverhältnisse fort, bei zuvor arbeitslosen Frauen galt das für $66 \%$.

Über den Beobachtungszeitraum hinweg wirken die Verläufe trotz einiger Schwankungen recht stabil. Etwa seit dem Jahr 2002 zeigt sich für Frauen insgesamt ein leichter Anstieg der Beschäftigungsstabilität, dies gilt aber nicht für Frauen mit vorheriger Arbeitslosigkeit. Bei den Männern sind leichte Rückgänge der Sechs- und Zwölf-Monats-Verbleibswahrscheinlichkeiten in neu begonnenen Beschäftigungen zu verzeichnen, auch wenn diese bei der Gruppe der vorher Arbeitslosen zuletzt wieder ansteigen. Begründungen für die insgesamt hohe Beschäftigungsstabilität in der Krise 2008/2009 könnten sein, dass einerseits Unternehmen verstärkt an ihrer Belegschaft festhielten, auch um teilweise bereits drohendem Fachkräftemangel entgegenzuwirken (vgl. Möller 2010; Burda/Hunt 2011). Andererseits waren Beschäftigte vermutlich seltener bereit, von sich aus zu kündigen, weil das Risiko von Arbeitslosigkeit oder Langzeitarbeitslosigkeit und die damit einhergehenden Kosten seit den Hartz-Reformen höher eingeschätzt wurden (vgl. Erlinghagen 2010) bzw. die zu erzielenden Lohngewinne angesichts der gesunkenen Einstiegslöhne eher gering waren.

Mit multivariaten Modellen wurde der Frage nachgegangen, inwieweit sich die Beschäftigungsdauern zuvor arbeitsloser Personen während oder nach den Hartz-Reformen verändert haben, wenn dabei Veränderungen in der Merkmalsstruktur der Beschäftigten berücksichtigt werden. Darüber hinaus wurde untersucht, ob sich empirisch ein Zusammenhang zwischen der Dauer der vorangegangen Arbeitslosigkeit und der Dauer der anschließenden Beschäftigung zeigt und sich daher im Zuge der Hartz-Reformen die Stabilität der Beschäftigung etwa von zuvor Langzeitarbeitslosen verändert hat.

Die Schätzungen werden getrennt nach Geschlecht durchgeführt. Wie bei den Lohnschätzungen wird die Entwicklung der Beschäftigungsdauern in unterschiedlichen Perioden des Beobachtungszeitraums mit Dummyvariablen abgebildet. Tabelle 3 enthält als Auszug die im Vordergrund des Interesses stehenden Schätzergebnisse. ${ }^{7}$ Geschätzt werden Einflüsse der unabhängigen Variablen auf das Risiko, die Beschäftigung zu beenden. Die geschätzten Effekte werden in Form von Hazard Ratios angegeben. Bei binären und kategorischen Variablen sind die Ha-
ABB. 3

Verbleibswahrscheinlichkeiten in neu begonnenen sozialversicherungspflichtigen Beschäftigungsverhältnissen 1998 - 2009 in Westdeutschland*
- 6 Monate
12 Monate
6 Monate, vorher arbeitslos
12 Monate, vorher arbeitslos

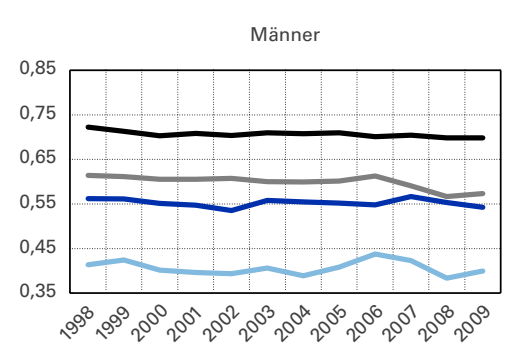

Frauen

*insgesamt und nach Arbeitslosigkeit.

Quelle: IEB; Berechnungen der Autoren

TABELLE 3

\section{Determinanten und Effekte unterschiedlicher Zeiträume auf die Beschäftigungsdauer nach Arbeitslosigkeit, Westdeutschland}

\begin{tabular}{lcc}
\hline & Männer & Frauen \\
\hline Periode (Ref. 1998-2002) & & \\
\hline $2003-2005$ & $0,934^{* * *}$ & 0,981 \\
$2006-2008$ & $0,951^{* * *}$ & 0,976 \\
\hline Dauer der Arbeitslosigkeit (Referenzkategorien bis 6 Monate) & & \\
\hline 6 bis 9 Monate & $1,083^{* * *}$ & 0,989 \\
\hline bis 12 Monate & $1,082^{* * *}$ & 0,978 \\
\hline 12 bis 24 Monate & $1,062^{* * *}$ & 0,978 \\
\hline 24 Monate und mehr & $1,255^{* * *}$ & $1,083^{* *}$ \\
\hline Varianz Frailty Term (log 0) & $-0,893^{* * *}$ & $-2,218^{* * *}$ \\
\hline Anzahl der Beobachtungen & 72.031 & 48.056 \\
\hline
\end{tabular}

Hazard Ratios, Signifikanzniveau: * $10 \%, * * 5 \%$ und *** $1 \%$.

Weitere Kontrollvariablen: Qualifikation, Alter, Ausländer, Teilzeit, Betriebsgröße, Wirtschaftszweig regionale Arbeitsnachfrage, Bundesland, Eintrittsquartal.

Quelle: IEB; Statistisches Bundesamt; Berechnungen der Autoren.

6 Abbildung 3 liegen getrennt nach Jahr der Beschäftigungsaufnahme durchgeführte Kaplan-Meier-Schätzungen zugrunde. Die jeweiligen Wahrscheinlichkeiten, nach sechs und zwölf Monaten noch beschäftigt zu sein, wurden für alle neu begonnenen sozialversicherungspflichtigen Beschäftigungen und für die Untergruppe der Beschäftigungen im Anschluss an eine Arbeitslosigkeit berechnet.

7 Ausführliche Schätzergebnisse sowie zusätzliche interagierte Schätzungen sind bei den Autoren erhältlich. 
zard Ratios gleich dem Quotienten aus den Hazardraten für die jeweilige Gruppe und die Referenzgruppe. Werte der Hazard Ratio größer als 1 deuten darauf hin, dass die Variable das Risiko der Beendigung der Beschäftigung erhöht und damit auf die Beschäftigungsdauer einen negativen Einfluss ausübt. Werte kleiner als 1 implizieren längere Verweildauern in Beschäftigung als in der Referenzkategorie.

Es zeigt sich für Männer im Vergleich zum Referenzzeitraum (1998-2002) sowohl für die Reformperiode (2003 - 2005) als auch für die Zeit danach (2006 - 2008) ein signifikanter Anstieg der Beschäftigungsstabilität im Anschluss an eine Arbeitslosigkeitsphase. Für Frauen sind die geschätzten Einflüsse schwächer und nicht signifikant.

Bezüglich der Dauer der vorangegangenen Arbeitslosigkeit werden weiterhin für Männer für alle Dauerkategorien signifikante Hazard Ratios größer 1 geschätzt und damit negative Effekte auf die Dauer der nachfolgenden Beschäftigung im Vergleich zur Referenzgruppe. Am stärksten ist der Effekt für die Gruppe der Männer mit vorangegangener Dauer der Arbeitslosigkeit von 24 Monaten und mehr. Für Frauen zeigt sich für sehr lange Arbeitslosigkeitsdauern von mehr als 24 Monaten ebenfalls ein solcher Effekt, während bei kürzeren Arbeitslosigkeitsdauern keine signifikanten Unterschiede zur Referenzgruppe geschätzt werden. Insgesamt erscheint aufgrund der meist nur schwach oder nicht signifikanten Koeffizienten der Zusammenhang zwischen Dauer der vorhergehenden Arbeitslosigkeit und Beschäftigungsdauer bei Frauen deutlich schwächer ausgeprägt zu sein als bei Männern.

In weiteren Schätzungen wurden alle erklärenden Variablen mit den Reformperioden interagiert, um Veränderungen im Zusammenhang zwischen Merkmalen und Beschäftigungsdauer über die Zeit zu untersuchen (siehe Fußnote 7). Die Ergebnisse sind uneinheitlich, weisen jedoch bei langzeitarbeitslosen Männern und bei Frauen mit Arbeitslosigkeitsdauern von über 24 Monaten auf kürzere Beschäftigungsdauern im neuen Arbeitsverhältnis während und nach den Hartz-Reformen hin. Dies könnte auf eine höhere Konzessionsbereitschaft hindeuten, die dann zur Aufnahme einer suboptimalen und wenig stabilen Beschäftigung führte.

Der Einfluss der übrigen Kontrollvariablen ist weitgehend plausibel. Allerdings zeigt sich, dass für bestimmte Personengruppen und bestimmte Beschäftigungsverhältnisse deutliche Unterschiede in den Beschäftigungsdauern bestehen. So steigt mit zunehmender Qualifikation, höherem Lebensalter und in größeren Betrieben die Beschäftigungsstabilität. Nach Wirtschaftszweigen hebt sich erneut der Bereich der Arbeitnehmerüberlassung ab, für den ein um ein Vielfaches höheres Risiko der Beendigung des Beschäftigungsverhältnisses und damit weit kürzere Beschäftigungsdauern als in den anderen Wirtschaftszweigen geschätzt werden. Auch ausländische Beschäftigte und teilzeitbeschäftigte Männer haben im Vergleich zur jeweiligen Referenzgruppe instabilere Beschäftigungsverhältnis- se. Bei Frauen sind Teilzeitbeschäftigungen dagegen etwas stabiler als Vollzeitstellen, was dafür spricht, dass diese Regelung häufig gewünscht ist, wenn auch möglicherweise aufgrund mangelnder Betreuungsalternativen für Kinder oder andere Familienangehörige. ${ }^{8}$

\section{Zusammenfassung}

Wir haben anhand der Stabilität und Entlohnung neuer sozialversicherungspflichtiger Beschäftigungsverhältnisse untersucht, wie sich die Beschäftigungsqualität im Anschluss an eine Arbeitslosigkeit seit Einführung der HartzReformen verändert hat.

Zunächst konnte ein kräftiger Rückgang der Reallöhne in neu begonnenen Vollzeitbeschäftigungsverhältnissen und insbesondere bei vorangegangener Arbeitslosigkeit sowie eine zunehmende Spreizung der Verteilung der Einstiegslöhne in Westdeutschland belegt werden. Für die Jahre 2006 bis 2009 zeigte sich für zuvor arbeitslose Männer und Frauen bei Berücksichtigung einer Vielzahl von individuellen, regionalen und betrieblichen Einflüssen bei neuen Beschäftigungsverhältnissen ein Lohnrückgang von mehr als $10 \%$ gegenüber dem Zeitraum vor den Hartz-Reformen. Darüber hinaus wurden für einzelne Gruppen innerhalb der untersuchten Personen mit Arbeitslosigkeit vor der Beschäftigung deutlich niedrigere Löhne geschätzt: Personen mit längerer Dauer der Arbeitslosigkeit und besonders Langzeitarbeitslose, Geringqualifizierte und Personen in Zeitarbeit.

Hinsichtlich der Beschäftigungsdauern sind unsere Befunde weniger eindeutig. Deskriptiv zeigt sich für die Zeit von 1998 bis 2009, dass die Beschäftigungsdauern in neuen Jobs nur bei Männern und nur leicht zurückgegangen sind. In multivariaten Analysen wurden aber zum Teil auch auf längere Beschäftigungsdauern hindeutende Periodeneinflüsse geschätzt. Ein klareres Ergebnis ist, dass im Vergleich zu anderen Personen zuvor Arbeitslose im neuen Job deutlich kürzere Beschäftigungsdauern haben (siehe auch Giannelli et al. 2013). Dabei zeigten sich für Langzeitarbeitslose, aber auch für Geringqualifizierte und Leiharbeiter noch einmal kürzere Beschäftigungsdauern.

Zum Teil dürften die insgesamt eher stabilen Beschäftigungsdauern auch auf die negative Entwicklung bei der Entlohnung zurückzuführen sein. Gründe für stabile Beschäftigungsverhältnisse liegen zum einen in der Angst vor erneuter Arbeitslosigkeit und dem damit verbundenen Statusverlust, zum anderen sind die möglichen Wechseloptionen aufgrund der geringen Löhne häufig wenig interessant. Das führt dazu, dass vormals Arbeitslose wie auch andere

8 Zu den Determinanten des Übergangs von Frauen in Teilzeitbeschäftigung siehe auch Vogel (2009). 
Beschäftigte eine einmal angenommene Stelle lieber etwas länger halten, als auf eine unsichere Stelle mit erneuter Probezeit, die zudem noch schlechter bezahlt ist, zu wechseln.

Den hier vorgestellten Ergebnissen nach haben Personen, die aus Arbeitslosigkeit heraus eine neue Beschäftigung aufnehmen, deutlich geringere Löhne und auch kürzere Beschäftigungsdauern. Bei ohnehin im Zeitablauf sinkenden Reallöhnen in neuen Beschäftigungsverhältnissen werden mit zunehmender Arbeitslosigkeitsdauer noch einmal schlechter bezahlte Beschäftigungen aufgenommen. Darüber hinaus werden für langzeitarbeitslose Männer und bei sehr langen Arbeitslosigkeitsdauern für Frauen in einzelnen Perioden zusätzliche Lohnabschläge geschätzt. Auf die Beschäftigungsdauer wirkt sich vor allem bei Männern die Dauer der vorangegangenen Arbeitslosigkeit verkürzend aus. Damit ist im Untersuchungszeitraum innerhalb der Gruppe zuvor arbeitsloser Personen, trotz zwischenzeitlicher Einführung der Hartz-Reformen, die Benachteiligung von Langzeitarbeitslosen hinsichtlich Beschäftigungsdauer und Entlohnung keinesfalls geringer geworden.

\section{LITERATUR}

Burda, M./Hunt, J. (2011): What explains the German labor market miracle in the great recession? Brookings Papers on Economic Activity, S. 273-319 Dustmann, C./Ludsteck, J./Schönberg, U. (2009): Revisiting the German wage structure, in:The Quarterly Journal of Economics 124 (2), S. 843-881 Erlinghagen, M. (2010): Mehr Angst vor Arbeitsplatzverlust seit Hartz? Langfristige Entwicklung der Beschäftigungsunsicherheit in Deutschland: Universität Duisburg-Essen, IAQ-Report (02)

Fahr, R./Sunde, U. (2009): Did the Hartz Reforms speed-up the matching process? A macro-evaluation using empirical matching functions, in: German Economic Review 10 (3), S. 284-316

Gartner, H./Klinger, S. (2010): Verbesserte Institutionen für den Arbeitsmarkt in der Wirtschaftskrise, in: Wirtschaftsdienst 90 (11), S. 728-734

Goebel, J./Richter, M. (2007): Nach der Einführung von Arbeitslosengeld II: Deutlich mehr Verlierer als Gewinner unter den Hilfeempfängern, DIW-Wochenbericht 50/2007, Berlin

Giannelli, G. C./Jaenichen, U./Rothe, T. (2013): Doing well in reforming the labour market? Recent trends in job stability and wages in Germany, IZA Discussion Paper (7580), Bonn

Hertweck, M. S./Sigrist, O. (2012): The aggregate effects of the Hartz Reforms in Germany: Universität Konstanz, Working Paper Series of the Department of Economics (38), Konstanz

Klinger, S./Rothe, T. (2012): Der Rückgang der Langzeitarbeitslosigkeit in Deutschland: Ein Erfolg der Hartz-Reformen oder konjunktureller Effekt? in: Schmollers Jahrbuch 132 (1), S. 89-121
Klinger, S./Rothe, T./Weber, E. (2013): Makroökonomische Perspektive auf die Hartz-Reformen: Die Vorteile überwiegen, IAB-Kurzbericht (11), Nürnberg Klinger, S./Weber, E. (2012): Decomposing Beveridge curve dynamics by correlated unobserved components, IAB Discussion Paper (28), Nürnberg Koller, L./Rudolph, H. (2011): Arbeitsaufnahmen von SGB-II-Leistungsempfängern: Viele Jobs von kurzer Dauer, IAB Kurzbericht (14), Nürnberg Krause, M. U./Uhlig, H. (2012): Transitions in the German labor market: Structure and crisis, in: Journal of Monetary Economics 59 (1), S. 64-79 Krebs, T./Scheffel, M. (2013): Macroeconomic evaluation of labor market reforms in Germany, Internationaler Währungsfond, IMF Working Paper 13/42, Washington D.C.

Launov, A./Wälde, K. (2013a): Thumbscrew for agencies or for individuals? How to reduce unemployment, working paper, August 2013, www.waelde.com Launov, A./Wälde, K. (2013b): Estimating incentive and welfare effects of nonstationary unemployment benefits, in: International Economic Review 54 (4), S. $1159-1198$

Möller, J. (2010): The German labor market response in the world recession. De-mystifying a miracle, in: Zeitschrift für ArbeitsmarktForschung 42 (4), S. $325-336$

Rebien, M./Kettner, A. (2011): Die Konzessionsbereitschaft von arbeitslosen Bewerbern und Beschäftigten nach den Hartz-Reformen, in: WSI-Mitteilungen 64 (5), S. 218-225, http://www.boeckler.de/wsi_34969_34981.htm

Riphahn, R. T./Schnitzlein, D. D. (2011): Wage mobility in East and West Germany, IZA Discussion Paper (6246), Bonn

Rothe, T. (2009): Arbeitsmarktentwicklung im Konjunkturverlauf: Nicht zuletzt eine Frage der Einstellungen, IAB-Kurzbericht (13), Nürnberg

Vogel, C. (2009): Teilzeitbeschäftigung: Ausmaß und Bestimmungsgründe der Erwerbsübergänge von Frauen, in: Zeitschrift für ArbeitsmarktForschung $42(2)$, S. $170-181$

\section{AUTOREN}

URSULA JAENICHEN, Dr., ist wissenschaftliche Mitarbeiterin im Kompetenzzentrum Empirische Methoden (KEM) des Instituts für Arbeitsmarkt- und Berufsforschung (IAB) der Bundesagentur für Arbeit. Arbeitsschwerpunkte: Mikroanalysen mit Prozessdaten der Bundesagentur für Arbeit.

ursula.jaenichen@iab.de

THOMAS ROTHE, Dr., ist wissenschaftlicher Mitarbeiter im Forschungsbereich Arbeitsmarktprozesse und Institutionen des IAB. Arbeitsschwerpunkte: Zugänge in und Abgänge aus Arbeitslosigkeit aus gesamtwirtschaftlicher Perspektive.

thomas.rothe@iab.de 